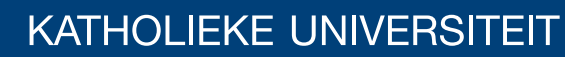 \\ LEUVEN
}

\section{Faculty of Business and Economics}

\section{KHLQAAYDORLCHUQVIISLREOP}

(]

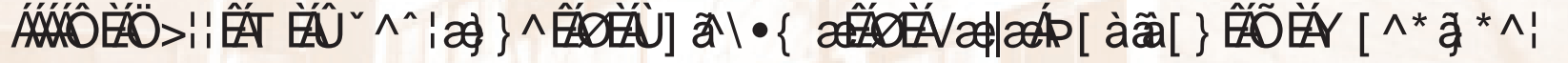

DEPARTMENT OF DECISION SCIENCES AND INFORMATION MANAGEMENT (KBI) 


\title{
The interval ordering problem
}

\author{
Christoph Dürr*, Maurice Queyranne ${ }^{\dagger}$, Frits C.R. Spieksma ${ }^{\ddagger}$, \\ Fabrice Talla Nobibon ${ }^{\ddagger}$, Gerhard J. Woeginger ${ }^{\S}$
}

\begin{abstract}
For a given set of intervals on the real line, we consider the problem of ordering the intervals with the goal of minimizing an objective function that depends on the exposed interval pieces (that is, the pieces that are not covered by earlier intervals in the ordering). This problem is motivated by an application in molecular biology that concerns the determination of the structure of the backbone of a protein.

We present polynomial-time algorithms for several natural special cases of the problem that cover the situation where the interval boundaries are agreeably ordered and the situation where the interval set is laminar. Also the bottleneck variant of the problem is shown to be solvable in polynomial time. Finally we prove that the general problem is NP-hard, and that the existence of a constant-factor-approximation algorithm is unlikely.
\end{abstract}

Keywords: dynamic programming; bottleneck problem; NP-hard; exposed part; agreeable intervals; laminar intervals.

\section{Introduction}

Let us consider a set $\mathcal{I}$ of $n$ intervals $I_{j}=\left[a_{j}, b_{j}\right)$ for $j=1,2, \ldots, n$ on the real line. The length of interval $I_{j}$ is denoted by $\left|I_{j}\right|=b_{j}-a_{j}$. As usual, the length of a union of disjoint intervals is the sum of the lengths of the individual intervals. For an interval $I_{j}$ and a subset $\mathcal{S} \subset \mathcal{I}$ of the intervals, we define $I_{j} \backslash \bigcup_{I \in \mathcal{S}} I$ to be that part of interval $I_{j}$ that is not covered by the union of the intervals in $\mathcal{S}$; throughout this text this uncovered part will be called the exposed part of $I_{j}$ relative to subset $\mathcal{S}$. Notice that the exposed part depends upon $\mathcal{S}$ and in general need not be an interval. (If the intervals in $\mathcal{I}$ are pairwise disjoint, then of course the exposed part of any interval $I$ relative to any set $\mathcal{S}$ of intervals not containing $I$ is the interval $I$ itself.)

We investigate an interval ordering problem that is built around a cost function $f$ that assigns to every interval of length $p$ a corresponding real cost $f(p)$. The cost of a set $\mathcal{S}$ of pairwise disjoint intervals is the sum of the costs of the individual intervals in $\mathcal{S}$. The cost of an ordering $\alpha=(\alpha(1), \alpha(2), \ldots, \alpha(n))$ of all $n$ intervals is the result of summing up in that order, for every interval, the cost of its exposed part with respect to the previous intervals. Formally, the problem is defined as follows.

${ }^{*}$ CNRS, LIX, Ecole Polytechnique, 91128 Palaiseau, France. durr@lix.polytechnique.fr

${ }^{\dagger}$ University of British Columbia. maurice.queyranne@sauder.ubc.ca

${ }_{\ddagger}^{\ddagger}$ Leuven University, Operations Research Group, Naamsestraat 69, B-3000 Leuven, Belgium. \{Frits.Spieksma;Fabrice.TallaNobibon\}@econ.kuleuven.be

$\S$ Technical University of Eindhoven. gwoegi@win.tue.nl 
Definition 1.1. The Interval Ordering Problem: Given a function $f: \mathbb{R} \rightarrow \mathbb{R}$ and $n$ intervals $I_{1}, \ldots, I_{n}$ over the real line, find an ordering $\alpha \in \Sigma_{n}$ such that the cost

$$
\sum_{k=1}^{n} f\left(\left|I_{\alpha(k)} \backslash \bigcup_{j=1}^{k-1} I_{\alpha(j)}\right|\right),
$$

is minimized, where $\Sigma_{n}$ denotes the set of all the permutations of $\{1,2, \ldots, n\}$.

Observe that the interval ordering problem becomes trivial, if all intervals are pairwise disjoint (since then all orderings yield the same cost). In the rest of this paper, an instance of the interval ordering problem is represented by $(\mathcal{I}, f)$ where $\mathcal{I}$ is the set of intervals and $f$ is the cost function.

Example 1.2. Consider the instance that consists of the five intervals $I_{1}=[0,1), I_{2}=[1,2)$, $I_{3}=[2,3), I_{4}=[3,6)$ and $I_{5}=[0,5)$, and the cost function $f(x)=2^{x}$. An optimal solution for this instance is given by the sequence $\alpha=(1,2,3,5,4)$ with a total cost of 12 .

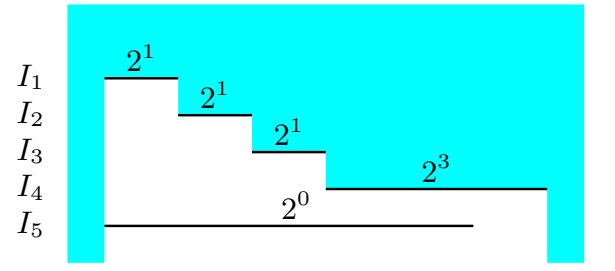

greedy ordering with respect to interval length

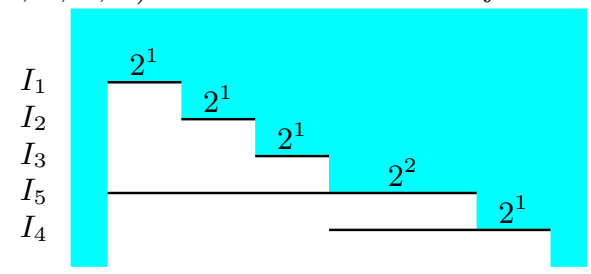

optimal ordering

This example illustrates that in general an optimal solution will not sequence the intervals in order of increasing length (and it can be verified that in Example 1.2 no such sequence can yield the optimal objective value). The next example illustrates that also the following natural greedy algorithm fails: "Always select the interval with the smallest exposed part relative to the intervals sequenced so far".

Example 1.3. Consider the instance that consists of $I_{1}=[2 k, 4 k+\epsilon), I_{2}=[k, 5 k+\epsilon)$, $I_{3}=[0,2 k), I_{4}=[4 k+\epsilon, 6 k+\epsilon)$ for some constants $k, \epsilon>0$ with the cost function $f(x)=2^{x}$. The greedy sequence $(3,4,1,2)$ achieves a cost of $2^{2 k+\epsilon}+2^{2 k+1}+2^{0}$, whereas the optimal solution $(1,2,3,4)$ has cost $2^{2 k+\epsilon}+2^{2 k}+2^{k+1}$. The ratio between both costs can be made arbitrarily close to 2, by choosing arbitrarily large $k$ and arbitrarily small $\epsilon>0$.

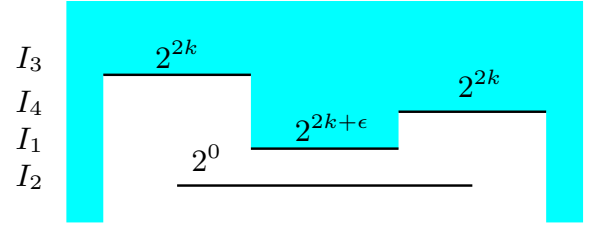

greedy ordering with respect to exposed length

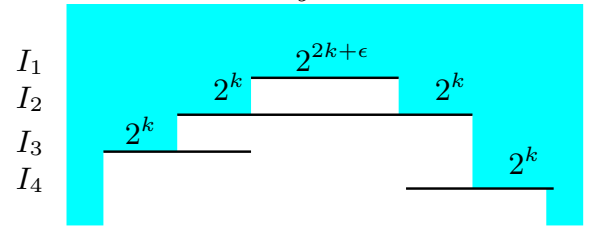

optimal ordering

The contributions of this paper are twofold: On the positive side, we describe polynomialtime algorithms for some natural and fairly general special cases of the problem. On the negative side, we establish the computational complexity (NP-hardness) and the inapproximability of the problem.

The paper is organized as follows. In Section 2, we describe the motivating real world application (in molecular biology) that stands behind the interval ordering problem. In 
Section 3, we formulate and resolve a number of special cases of the problem that can be solved in polynomial time. In Section 4, we present complexity and in-approximability results. We conclude in Section 5.

\section{Motivation}

The protein folding problem consists of computing the 3-dimensional structure of a protein. A protein is a huge molecule consisting of many different atoms linked together. Consider now a simplified version of this problem where we only want to determine the structure of the backbone of the protein, that is, we are interested in determining the position of the main string of atoms. The exact sequence of atoms is known, and different approaches are being used in practice to determine their spatial structure. One possibility is to use Nano Magnetic Resonance (NMR) to determine the distances between some pairs of atoms. The goal is then to reconstruct a folding that matches the measured distances. There are different algorithms that solve the problem in this setting, we refer to [2] for an overview.

Formally, the problem can be described as follows. Given is a chain of $m$ atoms, each having some unknown position $p_{i} \in \mathbb{R}^{3}$ for $1 \leq i \leq m$. We are given the distances between all pairs of atoms $i, j$ with $|i-j| \leq 3$, as well as distances between some pairs of atoms $i, j$ with $|i-j|>3$. The goal is to determine positions of the atoms $p_{i}^{\prime} \in \mathbb{R}^{3}$ for $1 \leq i \leq m$, that satisfy all given pairwise distances.

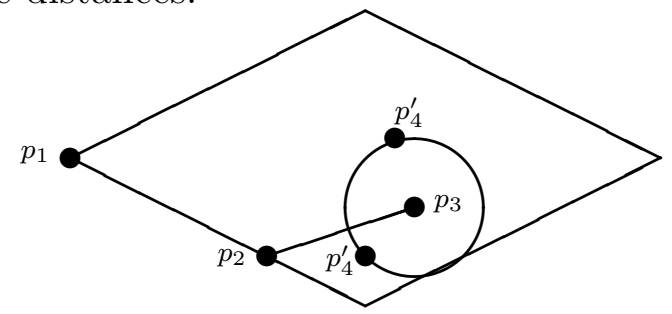

Figure 1: Four atoms and their positions in space. The circle is the intersection of balls centered respectively at $p_{2}$ and $p_{3}$. A further intersection with the ball centered at $p_{1}$, leaves two possibilities for atom 4 (identified by $p_{4}^{\prime}$ ), which are symmetric with respect to the hyperplane supported by $p_{1}, p_{2}, p_{3}$

We assume that no three successive atoms are collinear, meaning that the angle between two successive bonds $p_{i}-p_{i+1}, p_{i+1}-p_{i+2}$ is different from $\pi$. Under this assumption, the problem gets a combinatorial structure. Consider a chain of four atoms as in Figure 1. Without loss of generality we can place $p_{1}$ in the origin $(0,0,0)$, and $p_{2}$ in $(d, 0,0)$, where $d$ is the given distance between $p_{1}$ and $p_{2}$. Then $p_{3}$ can be placed in $(x, y, 0)$, for some coordinates $x, y$, determined by the distances with the first two atoms. By symmetry, without loss of generality we can assume $y \geq 0$ and even $y>0$ by the non-collinearity assumption. Now the possible positions for $p_{4}$ are at the intersection of the balls around $p_{1}, p_{2}, p_{3}$ with the radii given by the measured distances. The intersection of the balls around $p_{2}$ and $p_{3}$ is a circle with its center on the line through $p_{2}$ and $p_{3}$ (and on a plane, orthogonal to it). The intersection with the ball centered around $p_{1}$ results in at most two points, since by the non-collinearity assumption, $p_{1}$ does not lie on the latter line. These two points are mirrors 
of each other over the $p_{1}, p_{2}, p_{3}$ plane, and in the degenerate case we have a single point on this plane. Suppose - for this moment - that we only take the distances between atoms $i, j$ with $|i-j| \leq 3$ into account. Then, once the atoms 1,2,3 are fixed as described above, there are two possible positions for atom $4,2^{2}$ possible positions for atom $5, \ldots, 2^{m-3}$ possible positions for atom $m$. Each position is described by some binary string $x \in\{0,1\}^{m-3}$, that we index for convenience $x_{4}, x_{5}, \ldots, x_{m}$. The bit $x_{i}$ describes which of the two possible positions for atom $i$ is taken, relative to the atoms $i-3, i-2$, and $i-1$.

Up to now we have not used the known distances between atoms $i, j$ with $|i-j|>3$. Such a distance implies a constraint on the unknown binary string $x$. It enforces the bits $x_{i+4}, x_{i+5}, \ldots, x_{j}$ to those positions that yield a local folding such that atoms $i$ and $j$ are at the right distance. The problem now is to find, as efficiently as possible, values for the bits satisfying all measured distance constraints. Let us now state some notation to arrive at a formal definition of the problem.

Notation If $a>b$ then we denote by $[a, b]$ the empty interval. For any $a<b$ by $\{0,1\}^{[a, b]}$ we denote the set of bit strings of length $b-a+1$, and by convenience we do not index the bits from 1 on, but from $a$ to $b$. If $[a, b] \subseteq[c, d]$ and $x \in\{0,1\}^{[c, d]}$ then we denote by $x[a, b]$ the restriction of $x$ to the indices from $a$ to $b$. Also $\{0,1\}^{m}$ is a shortcut notation for $\{0,1\}^{[1, m]}$.

Definition 2.1. The BitString-Reconstruction Problem (BSRP): We are given an integer $m$, and $n$ triplets $\left(a_{i}, b_{i}, T_{i}\right)$ where $1 \leq a_{i}<b_{i} \leq m, T_{i}:\{0,1\}^{\left[a_{i}, b_{i}\right]} \rightarrow\{0,1\}$. The function $T_{i}$ is an oracle that returns 1 at a single element of the domain. The goal of the BSRP is to find a bit string $x \in\{0,1\}^{m}$, such that for all $i=1, \ldots, n$ we have $T_{i}\left(x\left[a_{i}, b_{i}\right]\right)=1$.

The idea is that a triplet in BSRP corresponds to a given distance between atoms $i$ and $j$ with $|i-j|>3$ in the folding problem. Formally, a triplet is defined by $(a=i, b=j, T)$ where $T$ is the boolean function, that accepts a bit string $z$ if and only if $z=x[a, b]$ for every bit string $x \in\{0,1\}^{m}$ describing atom positions where $i$ and $j$ are at the right distance. We assume that there is a unique bit string $z$ with this property. Already with this strong simplification, we are facing a non-trivial and interesting algorithmic problem.

A straightforward algorithm to solve BSRP employs a brute force approach: by letting $\xi$ be a symbol representing an unspecified bit, the idea of brute-force search is to start with a completely unspecified string $x=\xi^{n} \in\{0,1, \xi\}^{n}$, and to refine it using the distances between atoms $i$ and $j$ with $|i-j|>3$. More precisely:

The running time of the BruteForce search algorithm is $O\left(\sum_{i=1}^{n} 2^{\ell_{i}}\right)$. Clearly, this number depends on the order in which the triplets in the instance are presented to the algorithm. The only question remaining is in which order to process the given distances. In fact, it is our goal to find an order for the triplets in the instance of the BSRP to be passed to the BruteForce search algorithm in order to minimize the running time. This leads to the interval ordering problem that was described in the introduction with the following additional structure: (i) all data are integral, and (ii) the cost function $f$ is given by $f(x)=2^{x}$. 


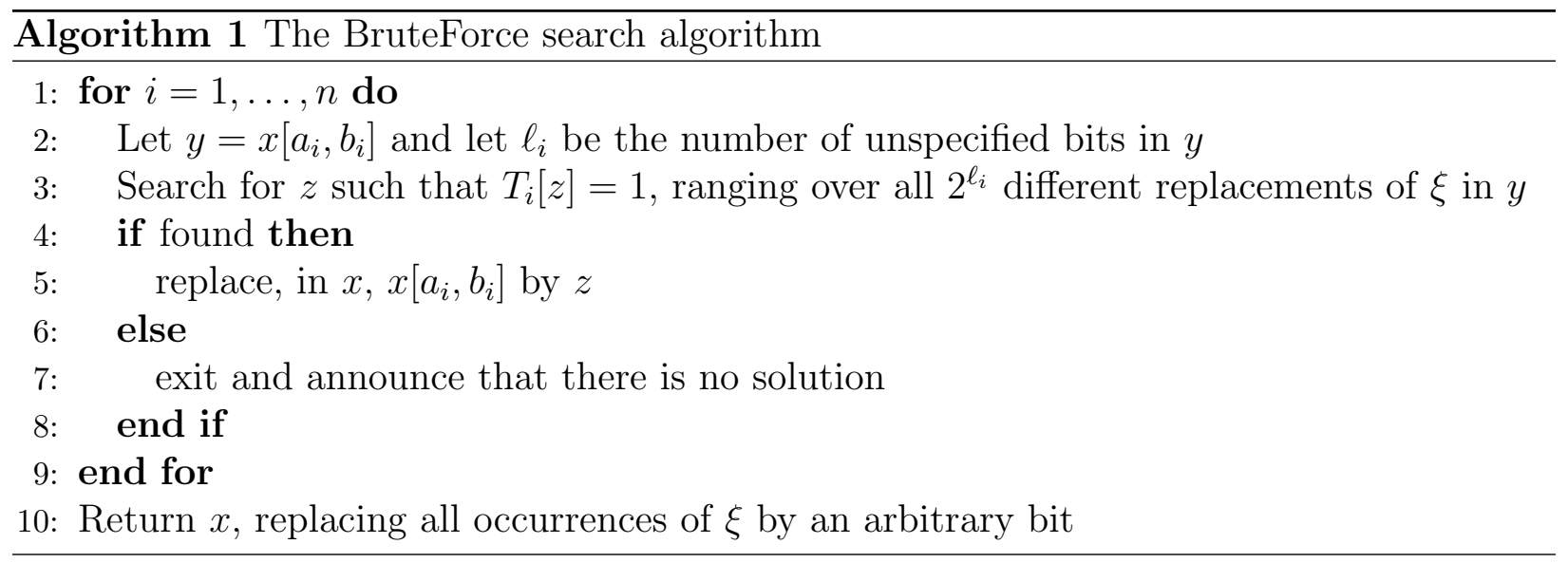

\section{Some polynomial time solvable cases}

In this section, we study some special cases of the interval ordering problem that can be solved in polynomial time. We first consider the case where the intervals are agreeable. We derive an $O\left(n^{3}\right)$ dynamic programming algorithm for solving this special case for any cost function $f$. When the cost function is continuous and convex, we propose a dynamic programming algorithm with time complexity $O\left(n^{2}\right)$. Next, we consider the case where the intervals are laminar and describe polynomial-time algorithms for solving the problem when the cost function $f$ is such that the function $g(x)=f(x)-f(0)$ is either super-additive or sub-additive. Finally, we study the bottleneck variant of the interval ordering problem and show that it can be solved in polynomial time when the cost function $f$ is either nondecreasing or non-increasing.

\subsection{Agreeable intervals}

We say that a set $\mathcal{I}$ of $n$ intervals $I_{i}=\left[a_{i}, b_{i}\right)$, for $i=1,2, \ldots, n$ is agreeable if there exists a permutation $\gamma$ of $\{1, \ldots, n\}$ such that $a_{\gamma(1)} \leq \ldots \leq a_{\gamma(n)}$ and $b_{\gamma(1)} \leq \ldots \leq b_{\gamma(n)}$. In other words, the ordering of the intervals induced by the left endpoints is the same as the ordering induced by the right endpoints. For ease of exposition, we will assume in the rest of this section that $\gamma$ is the permutation identity: thus we have $a_{1} \leq \ldots \leq a_{n}$ and $b_{1} \leq \ldots \leq b_{n}$. We can assume that two consecutive intervals $I_{i}$ and $I_{i+1}$ overlap (that is $a_{i+1}<b_{i}$ ) because otherwise this would split the problem into two sub-problems that can be solved independently. In what follows, we first consider the general case with an arbitrary cost function $f$, followed by a special case where the cost function $f$ is continuous and convex.

\subsubsection{Arbitrary cost function}

In this section, we consider instances $(\mathcal{I}, f)$ of the interval ordering problem with $\mathcal{I}$ agreeable and $f$ arbitrary. Observe that in the case of agreeable intervals, after selecting a first interval, the problem decomposes into (at most) two unrelated instances that are each agreeable; we will use this property to derive a dynamic programming algorithm.

For a formal definition of the decomposition, consider the set $\mathcal{I}=\left\{I_{1}, I_{i+1} \ldots, I_{n}\right\}$ of 
agreeable intervals. We consider the exposed parts of each of these intervals with respect to $\left\{I_{j}\right\}, 1 \leq j \leq n$. Since $\mathcal{I}$ is agreeable, the exposed parts are again intervals, and we distinguish between those before $I_{j}$ and those after $I_{j}$.

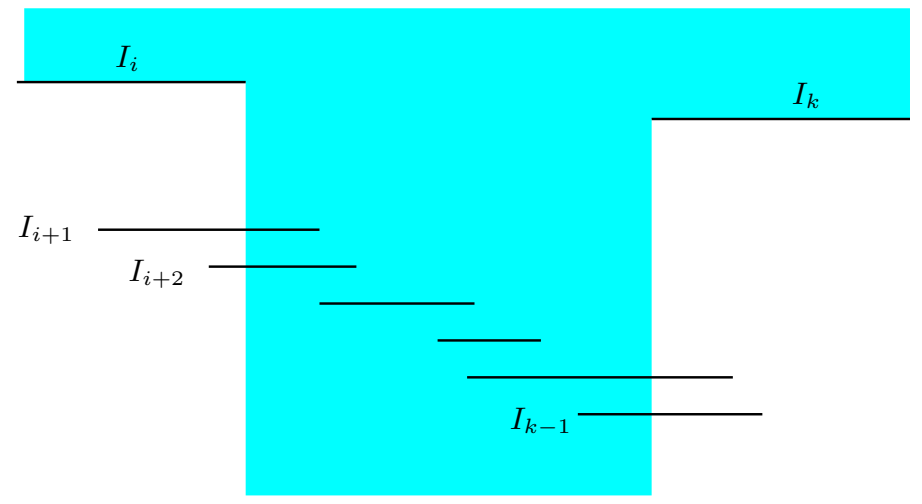

Figure 2: The subinstance $\mathcal{I}_{i, k}$.

For convenience define $b_{0}=a_{1}$ and $a_{n+1}=b_{n}$. For any pair of indices $0 \leq i, k \leq n+1$ we define the subinstance $\mathcal{I}_{i, k}:=\left\{I_{j} \cap\left[b_{i}, a_{k}\right): i<j<k\right\}$. Notice that if $b_{i} \geq a_{k}$, then $\mathcal{I}_{i, k}$ consists of $k-i-1$ intervals of zero length. Let $O(i, k)$ be the cost of an optimum solution to $\left(\mathcal{I}_{i, k}, f\right)$, where $O(i, k)=0$ if $\mathcal{I}_{i, k}=\emptyset$. We have the following recursion.

Lemma 3.1. For $0 \leq i<k \leq n+1$ we have $O(i, k)=0$ in case $i+1=k$ and otherwise

$$
O(i, k)=\min _{i<j<k}\left\{O(i, j)+f\left(\left|I_{j} \cap\left[b_{i}, a_{k}\right)\right|\right)+O(j, k)\right\} .
$$

Proof: The case $i+1=k$ follows from $\mathcal{I}_{i, i+1}=\{\}$ and the remaining case is immediate.

Theorem 3.2. The interval ordering problem $(\mathcal{I}, f)$ with $\mathcal{I}$ agreeable and $f$ arbitrary, can be solved in $O\left(n^{3}\right)$.

Proof: Lemma 3.1 leads to a dynamic programming algorithm with $O\left(n^{2}\right)$ variables, each computable in linear time.

\subsubsection{Continuous and convex cost function}

In this subsection, we still assume that the intervals in $\mathcal{I}$ are agreeable, but we consider the cost function $f$ to be continuous and convex. We need the following result, due to Karamata [4] (see also pages 30-32 in Beckenbach and Bellman [1]).

Lemma 3.3. Given are $2 q+2$ numbers $\left\{x_{k}, y_{k}\right\}, k=0,1, \ldots, q$ satisfying:

- $x_{0} \geq x_{1} \geq \ldots \geq x_{q}$, and $y_{0} \geq y_{1} \geq \ldots \geq y_{q}$,

- for each $k=0,1, \ldots, q-1: \sum_{i=0}^{k} x_{i} \geq \sum_{i=0}^{k} y_{i}$, and

- $\sum_{i=0}^{q} x_{i}=\sum_{i=0}^{q} y_{i}$. 
Then, for any continuous, convex function $f$ we have:

$$
\sum_{i=0}^{q} f\left(x_{i}\right) \geq \sum_{i=0}^{q} f\left(y_{i}\right)
$$

Let $(\mathcal{I}, f)$ be an instance of the interval ordering problem where $\mathcal{I}$ is agreeable and contains $n$ intervals $I_{i}=\left[a_{i}, b_{i}\right), i=1, \ldots, n$ and $f$ is continuous and convex. For a given solution to $(\mathcal{I}, f)$ (i.e., a sequence of intervals), we call an interval $I_{i}$ an E-interval if $a_{i}$ is contained in the exposed part of interval $I_{i}$ relative to the set of intervals sequenced before $I_{i}$ (in that solution). Given an integer $k, 1 \leq k \leq n$, let $\mathcal{I}_{k}$ be the set containing the intervals $I_{i}=\left[a_{i}, b_{i}\right)$ for $i=k, \ldots, n$ and let $O_{k}$ be the value of an optimal solution to the instance $\left(\mathcal{I}_{k}, f\right)$. Notice that this definition implies that $\mathcal{I}=\mathcal{I}_{1}$. Further, interval $I_{k}$ is an $E$-interval in any feasible solution to $\left(\mathcal{I}_{k}, f\right)$.

Lemma 3.4. Let $(\mathcal{I}, f)$ be an instance of the interval ordering problem with $\mathcal{I}$ agreeable and $f$ continuous and convex; and let $\mathcal{I}_{k}$ defined as above.

If, in an optimal solution to $\left(\mathcal{I}_{k}, f\right)$, interval $I_{k}$ is the only E-interval, then

$$
O_{k}=f\left(b_{k}-a_{k}\right)+\sum_{i=k+1}^{n} f\left(b_{i}-b_{i-1}\right)
$$

Otherwise, in this optimal solution to $\left(\mathcal{I}_{k}, f\right)$, let $I_{j}$ with $j>k$ be the first E-interval, i.e., the E-interval with minimal $a_{j}$.

If $a_{j} \leq b_{k}$ then $j=k+1$ and

$$
O_{k}=f\left(a_{k+1}-a_{k}\right)+O_{k+1}
$$

If $a_{j}>b_{k}$, then

$$
O_{k}=f\left(b_{k}-a_{k}\right)+\sum_{i=k+1}^{\ell} f\left(b_{i}-b_{i-1}\right)+f\left(a_{j}-b_{\ell}\right)+(j-\ell-1) f(0)+O_{j}
$$

where $I_{\ell}$ is the latest interval in $\mathcal{I}_{k}$ that satisfies $b_{\ell}<a_{j}$.

Proof: We will show that if interval $I_{k}$ is the only $E$-interval, then the optimal sequence to $\left(\mathcal{I}_{k}, f\right)$ is simply $(k, k+1, \ldots, n)$. Otherwise, if there is another $E$-interval $I_{j}$, where $I_{j}$ is the first $E$-interval with $j>k$, then the optimal sequence to $\left(\mathcal{I}_{k}, f\right)$ is the sequence of the solution to $\left(\mathcal{I}_{j}, f\right)$ followed by $k, k+1, \ldots, j-1$. See Figure 3 for illustration.

Case 1: Interval $I_{k}$ is the only $E$-interval. We show that $\alpha_{0}=(k, k+1, \ldots, n)$ is an optimal sequence to $\left(\mathcal{I}_{k}, f\right)$. The sequence $\alpha_{0}$ partitions $\left[a_{k}, b_{n}\right)$ into $n-k+1$ nonempty segments, defined by $S_{0}=\left[a_{k}, b_{k}\right), S_{i}=\left[b_{k+i-1}, b_{k+i}\right)$ for $i=1, \ldots, n-k$ (this is true since the intervals are agreeable). Let $\sigma$ be a permutation of $\{0,1, \ldots, n-k\}$ such that $\left|S_{\sigma(i)}\right| \geq\left|S_{\sigma(i+1)}\right|$ for $i=0,1, \ldots, n-k-1$; the permutation $\sigma$ orders the segments induced by $\alpha_{0}$ in non-increasing length. Now, let $\alpha$ be some sequence of intervals $\left(\alpha \neq \alpha_{0}\right)$ which does not feature another $E$-interval apart from interval $I_{k}$. Clearly, $\alpha$ partitions $\left[a_{k}, b_{n}\right)$ into 


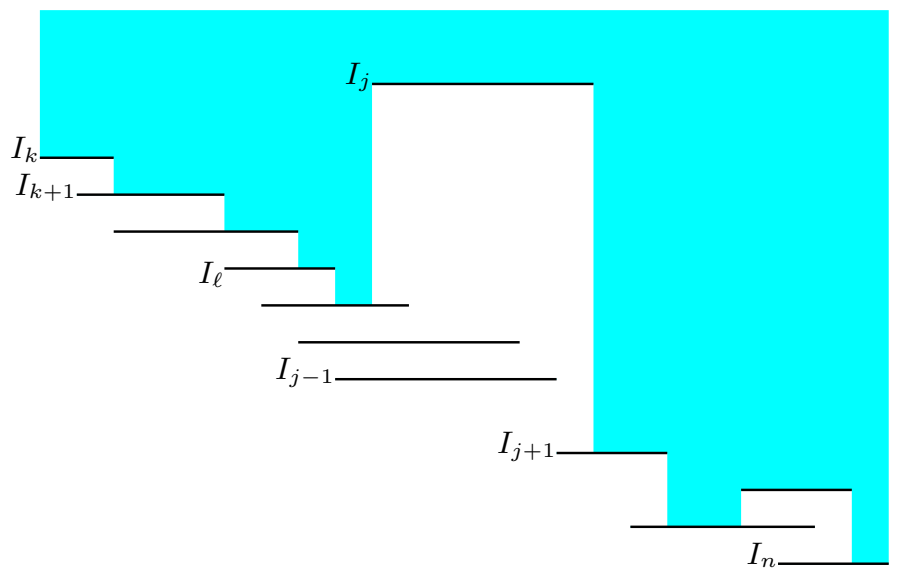

Figure 3: Recurrence relation of $O_{k}$. If $I_{j}$ is the first E-interval after $I_{k}$, then the cost divides into the cost of the intervals between $k$ and $j$, plus $O_{j}$

less than $n-k+1$ nonempty segments, each segment being defined by a pair from the set $\left\{a_{k}, b_{k}, b_{k+1}, \ldots, b_{n}\right\}$ (indeed, notice that the only way to have $n-k+1$ segments is when $\left.\alpha=\alpha_{0}\right)$. Let us suppose that $\alpha$ partitions $\left[a_{k}, b_{n}\right)$ into $p+1$ segments $(1 \leq p \leq n-k-1)$ $S_{0}^{\prime}, \ldots, S_{p}^{\prime}$ satisfying $\left|S_{0}^{\prime}\right| \geq\left|S_{1}^{\prime}\right| \geq \ldots \geq\left|S_{p}^{\prime}\right|$. For convenience set $S_{p+1}^{\prime}=\ldots=S_{m}^{\prime}=$ \{\} . A crucial observation is that because the segments are defined by points in the set $\left\{a_{k}, b_{k}, b_{k+1}, \ldots, b_{n}\right\}$, any segment $S_{i}^{\prime}(i=0,1, \ldots, p)$ is either identical to a segment $S_{j}$ for a given $j \in\{0,1, \ldots, n-k\}$ or is a union of consecutive intervals $S_{j}$. We will use this observation to argue that for each $m=0,1, \ldots, n-k+1$ :

$$
\sum_{i=0}^{m}\left|S_{i}^{\prime}\right| \geq \sum_{i=0}^{m}\left|S_{\sigma(i)}\right| .
$$

For any $m$ with $p \leq m \leq n-k$, we have that $\sum_{i=0}^{m}\left|S_{i}^{\prime}\right| \geq \sum_{i=0}^{m}\left|S_{\sigma(i)}\right|$. This is because $\cup_{i=0}^{m} S_{i}^{\prime}=\left[a_{k}, b_{n}\right)$ and $\cup_{i=0}^{m} S_{\sigma(i)} \subseteq\left[a_{k}, b_{n}\right)$ and the segments are disjoint.

We now show that (5) is also true for $m<p$, by induction on $m$. For the basis case, note that the the observation above immediately implies that $\left|S_{0}^{\prime}\right| \geq\left|S_{\sigma(0)}\right|$. For the induction step, we assume that $\sum_{i=0}^{m}\left|S_{i}^{\prime}\right| \geq \sum_{i=0}^{m}\left|S_{\sigma(i)}\right|$. The question now is whether

$$
\sum_{i=0}^{m+1}\left|S_{i}^{\prime}\right| \geq \sum_{i=0}^{m+1}\left|S_{\sigma(i)}\right|
$$

is true. Let us consider $S_{\sigma(m+1)}$. If each $S_{\sigma(r)}$ with $r \leq m$ is contained in the left-hand side of (6), then the validity of inequality (5) implies the validity of (6). Indeed, if $S_{\sigma(m+1)}$ is also contained in the left-hand side of (6), the inequality is certainly valid, else we know that $S_{m+1}^{\prime} \geq S_{\sigma(m+1)}$. If there exists an $S_{\sigma(r)}$ with $r \leq m$ not contained in the left-hand side of (6), then: $S_{m+1}^{\prime} \geq S_{\sigma(r)} \geq S_{\sigma(m+1)}$ (where the first inequality holds since the length of a segment $S_{\sigma(j)}$ not contained in the left-hand side of (6) is a lower bound for $S_{j}^{\prime}$ ).

We now invoke Lemma 3.3 by setting $q:=n-k+1$, and for $i=0,1, \ldots, n-k+1$ we set $x_{i}:=\left|S_{i}^{\prime}\right|, y_{i}:=\left|S_{i}\right|$. Clearly, the arguments given above imply that the conditions of 
Lemma 3.3 are satisfied. Hence, when $f$ is continuous and convex, the cost of $\alpha$ is greater than or equal to the cost of $\alpha_{0}$.

Case 2: There is another $E$-interval $I_{j}$, where $I_{j}$ is the first $E$-interval after $I_{k}$. For this case, we use the following observation. Let $I_{p}$ and $I_{q}$ be two consecutive intervals in a solution, and suppose that they are disjoint. Then it does not matter for the cost of the solution whether $I_{p}$ or $I_{q}$ is processed first of the two. Now since $I_{j}$ is an $E$-interval, it must be processed before all intervals $I_{i}$ that contain $a_{j}$ (otherwise $I_{j}$ is not an $E$-interval), and it can be processed before all intervals $I_{i}$ with $b_{i}<a_{j}$. Thus, we conclude that $I_{j}$ is processed before intervals indexed by $k, k+1, \ldots, j-1$. Since the intervals are agreeable, the exposed parts (after processing $I_{j}$ ) of the intervals before $I_{j}$ are disjoint with the intervals with index greater than $j$. Therefore we can assume that the intervals with index $k, \ldots, j-1$ are processed after the intervals with index $j, \ldots, n$. And of course, the latter intervals are processed optimally by a sequence of the solution to $\left(\mathcal{I}_{j}, f\right)$. Let $I_{\ell}$ with $\ell<j$ be the latest interval that does not intersect interval $I_{j}$. Notice that by the choice of $j$, the optimal sequence of the intervals $I_{k}, \ldots, I_{\ell}$ contains only one $E$-interval, namely $I_{k}$. Hence, that optimal sequence has a cost of $f\left(b_{k}-a_{k}\right)+\sum_{i=k+1}^{\ell} f\left(b_{i}-b_{i-1}\right)$. Finally, we need to take into account the intervals $I_{\ell+1}, \ldots, I_{j-1}$. Thus, we incur $f\left(a_{j}-b_{\ell}\right)$ for the exposed part between $b_{\ell}$ and $a_{j}$, corresponding to interval $I_{\ell+1}$, and we incur a cost of $f(0)$ for each of the remaining intervals. Notice that all intervals $I_{\ell+1}, \ldots, I_{j-1}$ are completely covered in this sequence. This completes the proof of this lemma.

Theorem 3.5. The interval ordering problem $(\mathcal{I}, f)$ with $\mathcal{I}$ agreeable and $f$ convex and continuous, can be solved in $O\left(n^{2}\right)$.

Proof: The $O\left(n^{2}\right)$-time complexity of the dynamic program following from Lemma 3.4 (see equations (2) and (3)) is explained by the fact that there are $n$ variables and each is a minimization over $O(n)$ values.

\subsection{Laminar intervals}

Let $(\mathcal{I}, f)$ be an instance of the interval ordering problem where $\mathcal{I}$ contains $n$ intervals $I_{i}=\left[a_{i}, b_{i}\right)$, for $i=1,2, \ldots, n$. We say that the set $\mathcal{I}$ of intervals is laminar if for any two intervals $I_{i}$ and $I_{j}$ in $\mathcal{I}$, either $I_{i} \cap I_{j}=\emptyset$ or one is included in the other. See Figure 4 for an illustration.

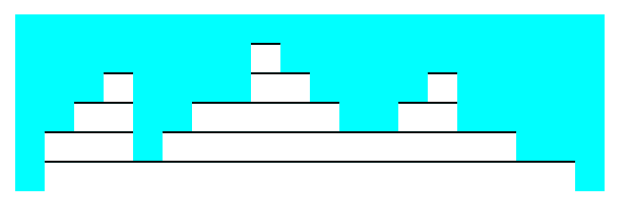

Figure 4: Illustration of laminar intervals

An ordering $\alpha$ respects the inclusions if for any two intervals $I_{i}$ and $I_{j}$ with $I_{i} \subsetneq I_{j}$ we have that $i$ appears before $j$ in $\alpha$. 
Lemma 3.6. Let $(\mathcal{I}, f)$ be an instance of the interval ordering problem with $\mathcal{I}$ laminar. If the function $g$ defined by $g(x)=f(x)-f(0)$ is super-additive i.e., $g(x+y) \geq g(x)+g(y)$ then any ordering that respects the inclusions is an optimal solution to $(\mathcal{I}, f)$.

Proof: Let $\alpha$ be an arbitrary order of optimal cost. We will show that there is another order respecting the inclusions and having a cost not greater than that of $\alpha$.

Suppose that $\alpha$ does not respect the inclusions. Then there is a pair $i, j$ with $I_{i} \subsetneq I_{j}$ and $j$ appears before $i$ in $\alpha$. Let $\alpha^{\prime}$ be the result of placing $j$ right after $i$ in the order $\alpha$. Let $x$ be the length of the exposed part of $I_{j}$ in $\alpha^{\prime}$, and $y$ be the length of the exposed part of $I_{i}$ in $\alpha^{\prime}$. Then $x+y$ is the length of the exposed part of $I_{j}$ in $\alpha$. Therefore the contribution of $I_{i}$ and $I_{j}$ to the cost of $\alpha$ is $f(x+y)+f(0)$ while their contribution to the cost of $\alpha^{\prime}$ is $f(x)+f(y)$.

Since $g$ is super-additive, it follows that $f(x+y)+f(0) \geq f(x)+f(y)$. We conclude that the cost of $\alpha^{\prime}$ is not more than the cost of $\alpha$. By repeating the argument, we eventually obtain an inclusion respecting order with optimal cost.

An inclusion respecting order can be found simply by sorting the intervals in increasing order of their lengths, breaking ties arbitrarily.

Theorem 3.7. The interval ordering problem $(\mathcal{I}, f)$ with $\mathcal{I}$ laminar and $f$ such that the function $g(x)=f(x)-f(0)$ is super-additive, can be solved in $O(n \log n)$ time

Proof: Immediate.

We show in Section 4 that the time-complexity of $O(n \log n)$ is actually best possible.

Remark 3.8. Notice that the problem $(\mathcal{I}, f)$ with $\mathcal{I}$ laminar and $f$ such that the function $g(x)=f(x)-f(0)$ is sub-additive, can also be solved in $O(n \log n)$ time by sorting the intervals in decreasing order of their lengths.

\subsection{Bottleneck variant of the interval ordering problem}

In this subsection, we consider the bottleneck variant of the interval ordering problem. Referring to the application described in Section 2, instead of looking for the exact complexity $O\left(\sum_{i=1}^{n} 2^{\ell_{i}}\right)$ of the BruteForce search algorithm, we focus on the maximum power of two that dominates this complexity. Hence, solving the bottleneck variant gives us a solution that is an approximation of the optimal solution to the interval ordering problem. The bottleneck variant is explicitly defined as follows.

Definition 3.9. The Bottleneck Interval Ordering Problem (BIO): Given a function $f$ and a set $\mathcal{I}=\left\{I_{1}, \ldots, I_{n}\right\}$ of intervals over the real line, find an ordering $\alpha \in \Sigma_{n}$ that minimizes the value

$$
\max _{k=1, \ldots, n} f\left(\left|I_{\alpha(k)} \backslash \bigcup_{j=1}^{k-1} I_{\alpha(j)}\right|\right) .
$$

A greedy algorithm for this variant would iteratively select the interval with the smallest exposed part. A formal description is given in Algorithm 2. In the rest of this section we prove that Algorithm 2 solves instances of BIO when the cost function $f$ is non-decreasing. Our proof is based on the following lemmas. 


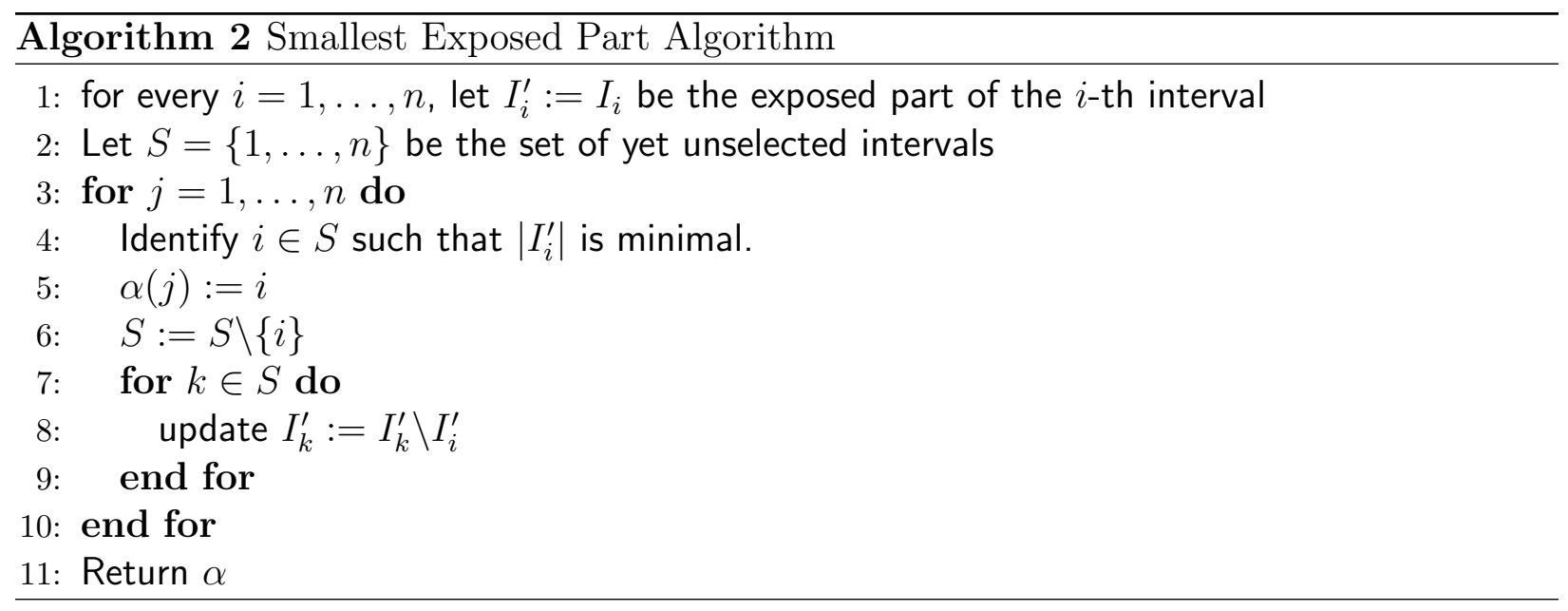

Lemma 3.10. Let $(\mathcal{I}, f)$ be an instance of BIO with a non-decreasing cost-function $f$. There exists an optimal solution to $(\mathcal{I}, f)$ starting with a smallest interval.

Proof: We prove this result by contradiction. Let $(\mathcal{I}, f)$ be an instance of BIO with a nondecreasing cost-function $f$. Assume that each optimal sequence to $(\mathcal{I}, f)$ does not start with a smallest interval. Consider an optimal sequence $\alpha=\left(\alpha(1), \ldots, \alpha\left(i_{0}\right), \ldots, \alpha(n)\right)$ to $(\mathcal{I}, f)$ with the corresponding optimal value $\operatorname{val}(\alpha)$. Clearly, $\operatorname{val}(\alpha) \geq f\left(\left|I_{\alpha(1)}\right|\right)$. Let $I_{\alpha\left(i_{0}\right)}$ be the first smallest interval in $\alpha$, i.e., $\left|I_{\alpha\left(i_{0}\right)}\right| \leq\left|I_{\alpha(j)}\right|$ for all $j \in\{1, \ldots, n\}$ and $\left|I_{\alpha\left(i_{0}\right)}\right|<\left|I_{\alpha(j)}\right|$ for all $j \in\left\{1, \ldots, i_{0}-1\right\}$. Consider now the sequence $\alpha^{\prime}=\left(\alpha\left(i_{0}\right), \alpha(1), \ldots, \ldots, \alpha(n)\right)$ where $\alpha\left(i_{0}\right)$ is move to the first position in $\alpha$. It is clear that this move only affects the intervals that were sequenced before $I_{\alpha\left(i_{0}\right)}$ in $\alpha$. Further, since $f$ is non-decreasing, and the length of each affected interval cannot have become larger, and $\left|I_{\alpha\left(i_{0}\right)}\right| \leq\left|I_{\alpha(1)}\right|$, we conclude that the objective value achieved by $\alpha^{\prime}$ does not exceed $\operatorname{val}(\alpha)$. Therefore, $\alpha^{\prime}$ is also an optimal sequence to $(\mathcal{I}, f)$, which is a contradiction.

Given an arbitrary instance $(\mathcal{I}, f)$ of $\mathrm{BIO}$ with $n$ intervals and $I_{i_{0}} \in \mathcal{I}$ a smallest interval, we define the $I_{i_{0}}$-reduced instance $\left(\overline{\mathcal{I}}_{i_{0}}, f\right)$ with $n-1$ intervals as follows. For any interval $I_{j} \in \mathcal{I}$

1. if $I_{j} \neq I_{i_{0}}$ and $I_{j} \cap I_{i_{0}}=\emptyset$ then $I_{j} \in \overline{\mathcal{I}}_{i_{0}}$;

2. if $I_{j} \neq I_{i_{0}}$ and $I_{j} \cap I_{i_{0}} \neq \emptyset$ then $I_{j} \backslash I_{i_{0}} \in \overline{\mathcal{I}}_{i_{0}}$.

Further, the real line is adapted accordingly such that $I_{j} \backslash I_{i_{0}}$ is an interval for all $j \neq i_{0}$.

Lemma 3.11. Let $(\mathcal{I}, f)$ be an instance of BIO with a non-decreasing cost-function $f$. Let $I_{i_{0}} \in \mathcal{I}$ be a smallest interval and $\alpha_{i_{0}}$ be an optimal sequence to $\left(\overline{\mathcal{I}}_{i_{0}}, f\right)$. Then $\left(i_{0}, \alpha_{i_{0}}\right)$ is an optimal sequence to $(\mathcal{I}, f)$.

Proof: Let $\operatorname{val}\left(\alpha_{i_{0}}\right)$ be the total cost of the optimal solution $\alpha_{i_{0}}$ to $\left(\overline{\mathcal{I}}_{i_{0}}, f\right)$.

1. If $f\left(\left|I_{i_{0}}\right|\right) \geq \operatorname{val}\left(\alpha_{i_{0}}\right)$ then $\left(i_{0}, \alpha_{i_{0}}\right)$ is clearly an optimal sequence to $(\mathcal{I}, f)$ (recall that $I_{i_{0}}$ is a smallest interval). 
2. On the other hand, suppose that $f\left(\left|I_{i_{0}}\right|\right)<\operatorname{val}\left(\alpha_{i_{0}}\right)$. Lemma 3.10 implies that there exists an optimal sequence $\alpha^{\prime}$ to $(\mathcal{I}, f)$ starting with $I_{i_{0}}$. After selecting $I_{i_{0}}$, the instance that remains is $\left(\overline{\mathcal{I}}_{i_{0}}, f\right)$, for which $\alpha_{i_{0}}$ is optimal. Therefore, $\left(i_{0}, \alpha_{i_{0}}\right)$ is an optimal solution to $(\mathcal{I}, f)$.

Theorem 3.12. The Bottleneck Interval Ordering problem $(\mathcal{I}, f)$ with $\mathcal{I}$ arbitrary and $f$ non-decreasing can be solved in $O\left(n^{2}\right)$.

Proof: The result follows from Lemma 3.10 and Lemma 3.11.

Remark 3.13. Notice that if the function $f$ is non-increasing then the instances of BIO with this cost function can be solved with an $O\left(n^{2}\right)$-time algorithm similar to Algorithm 2 where in line 6 instead of taking the interval with the smallest exposed part, we take the interval with the longest exposed part.

\section{Complexity results}

This section presents a number of negative results on the computational complexity of the interval ordering problem. Our first result shows that even the easy special cases discussed in Section 3.1 are not completely straightforward, and shows optimality of the algorithm given in Section 3.2.

Theorem 4.1. The interval ordering problem is at least as hard as the SORTING problem, even if (a) the intervals are agreeable, or if (b) the intervals form a laminar set. Consequently every algorithm for these special cases will have a time complexity of at least $\Omega(n \log n)$.

Proof: Let $x_{1}, \ldots, x_{n}$ be an arbitrary sequence of positive real numbers that form an instance of the SORTING problem. We construct a corresponding instance of the interval ordering problem that consists of the intervals $I_{j}=\left[0, x_{j}\right)$ for $j=1, \ldots, n$, together with the cost function $f(x)=2^{x}$. Note that this set of intervals is agreeable and laminar.

Note that the cost function $f(x)$ is such that $g(x)=f(x)-f(0)$ is super-additive on the positive real numbers. This observation easily yields that the optimal ordering of the intervals must sequence them by increasing right endpoint, and hence induces a solution to the SORTING problem.

Next, we will discuss the computational complexity of the interval ordering problem. We will show that there is little hope for finding a polynomial-time algorithm for solving the interval ordering problem in general. The reduction is from the following variant of the NP-hard PARTITION problem [3, problem SP12].

Instance: A finite set $\left\{q_{1}, q_{2}, \ldots, q_{n}\right\}$ of $n$ positive integers with sum $2 Q$; an integer $k$.

Question: Does there exists an index set $J \subseteq\{1, \ldots, n\}$ with $|J|=k$, such that $\sum_{j \in J} q_{j}=$ $\sum_{j \notin J} q_{j}=Q ?$ 
Lemma 4.2. Let $I$ be an instance of PARTITION and $N$ be an integer such that $2^{N-1}>$ $2^{n} Q+k$. Then there exists an instance $(\mathcal{I}, f)$ of the interval ordering problem that can be built from I in polynomial time and that satisfies the following conditions:

(i) If I is a YES-instance of PARTITION, then the total cost of an optimal sequence to $(\mathcal{I}, f)$ is at most $2^{n} Q+n-k$.

(ii) If I is a NO-instance of PARTITION, then the total cost of an optimal sequence to $(\mathcal{I}, f)$ is at least $2^{N}+2^{n} Q+n-k$.

Proof: Consider an arbitrary instance $I$ of PARTITION. We build the instance $(\mathcal{I}, f)$ of the interval ordering problem as follows. The cost function $f: \mathbb{N} \rightarrow \mathbb{N}$ is defined by $f(x)=0$ if $x$ is a power of two, and by $f(x)=x$ otherwise. The set $\mathcal{I}$ consists of $n+2$ intervals. First, for $i=1, \ldots, n$ there is a so-called element-interval of length $\ell_{i}=2^{n} q_{i}+1$. These element-intervals are pairwise disjoint and put back to back, so that they jointly cover the interval from 0 to $L:=\sum_{i=1}^{n} \ell_{i}=2^{n+1} Q+n$. Secondly, there is a so-called dummy-interval of length $\ell_{n+1}=2^{N}-2^{n} Q-k$ that goes from $L$ to $L+\ell_{n+1}$. Thirdly, there is the so-called main-interval that covers all other intervals, and that goes from 0 to $L+\ell_{n+1}$; hence the length of the main-interval is $2^{N}+2^{n} Q+n-k$. Clearly, this construction of $(\mathcal{I}, f)$ can be done in polynomial time. Next, we prove $(i)$ and $(i i)$.

(i) Assume that $I$ is a YES-instance of PARTITION, and let $J \subseteq\{1, \ldots, n\}$ be the corresponding index set. First select the element-intervals that correspond to the $q_{i}$ with $i \notin J$, then the main-interval, followed by the remaining element-intervals, and finally the dummy-interval. For the first batch of element-intervals we pay a cost of $2^{n} Q+n-k$. The exposed part of the main-interval then has length $2^{N}$, which yields a cost of 0 . This reduces the exposed part of all remaining intervals down to length 0 . The overall total cost is then $2^{n} Q+n-k$.

(ii) Now assume that $I$ is a NO-instance of PARTITION. We claim that in this case no sequencing can ever turn the length of the exposed part of the main-interval into a power of 2. Then the total cost is proportional to the total length, and hence at least $2^{N}+2^{n} Q+n-k$. It remains to prove the claim. We distinguish two cases. The first case deals with the time before the dummy-interval is sequenced. At such a point in time the length of the exposed part of the main-interval equals the length of the dummy-interval plus the length of the currently unsequenced element-intervals. The length of the dummy-interval is $2^{N}-2^{n} Q-k>2^{N-1}$. The length of the dummy interval plus the length of all elementintervals is $2^{N}+2^{n} Q+n-k<2^{N+1}$. Hence the only candidate power of 2 would be $2^{N}$. But in this case the subset of the element-intervals would have a total length of $2^{n} Q+n-k$, which would correspond to a solution to the PARTITION instance $I$; a contradiction. The second case deals with the time after the dummy-interval has been sequenced. At such a point in time the length of the exposed part of the main-interval equals the length of the remaining unsequenced element-intervals. But the total length of such a subset can never be a power of 2 .

Of course, Lemma 4.2 immediately yields the NP-hardness of the interval ordering problem. We will also deduce in-approximability from it. Suppose for the sake of contradiction 
that there is a polynomial-time approximation algorithm with some finite worst-case guarantee $\theta$. Pick an arbitrary instance $I$ of PARTITION, and choose the integer $N$ sufficiently large so that

$$
2^{N}>(\theta-1)\left(2^{n} Q+n-k\right) .
$$

Then $N$ is roughly $n+\log Q+\log \theta$, and hence its length is polynomially bounded in the size of instance $I$. We construct the instance $(\mathcal{I}, f)$ of the interval ordering problem as indicated in the proof of Lemma 4.2, and feed it into the approximation algorithm. The answer allows us to decide in polynomial time whether instance $I$ is a YES-instance or a NO-instance of PARTITION.

Theorem 4.3. The interval ordering problem is NP-hard. Furthermore, the interval ordering problem does not possess any polynomial-time approximation algorithm with constant worst-case guarantee, unless $P=N P$.

\section{Conclusion}

This text studies the problem of ordering a given set of intervals on the real line to minimize the total cost, where the cost incurred for an interval depends on the length of its exposed part when it is processed. We were motivated to consider this problem by an application in molecular biology. Our work proposes polynomial-time algorithms for some special cases of the problem. Further, we prove that the problem is NP-hard and it is unlikely to derive a constant-factor-approximation algorithm for solving the problem.

An important research direction that might be pursed in the future is the study of the complexity of the problem for the cost function $f(x)=2^{x}$ (note that our NP-hardness construction does not yield anything for this particular cost function). Finally, it would be interesting to see other special cases that can be solved in polynomial time.

Acknowledgements. Gerhard Woeginger has been supported by the Netherlands Organization for Scientific Research (NWO), grant 639.033.403, by DIAMANT (an NWO mathematics cluster), and by BSIK grant 03018 (BRICKS: Basic Research in Informatics for Creating the Knowledge Society).

\section{References}

[1] E. F. Beckenbach and R. Bellman. Inequalities. Springer-Verlag, 3rd printing, 1971.

[2] N. Maculan C. Lavor, L. Liberti. An overview of distinct approaches for the molecular distance geometry problem. Encyclopedia of Optimization, 2nd Edition, edited by $P$. Pardalos and C. Floudas, pages 1305-2311, 2008.

[3] M.R. Garey and D.S. Johnson. Computers and intractability: A guide to the theory of NP-completeness. W.H. Freeman and Co., 1979. 
[4] J. Karamata. Sur une inégalité relative aux fonctions convex. Publ. Math. Univ. Belgrade, 1:145-148, 1932. 